\title{
Technical feasibility of line-assisted complete closure technique for large mucosal defects after colorectal endoscopic submucosal dissection
}

\section{(9)( $\circledast \odot$}

\author{
Authors \\ Minoru Kato, Yoji Takeuchi, Yasushi Yamasaki, Masamichi Arao, \\ Sho Suzuki, Taro Iwatsubo, Kenta Hamada, Yusuke Tonai, Satoki \\ Shichijo, Noriko Matsuura, Hiroko Nakahira, Takashi Kanesaka, \\ Tomofumi Akasaka, Noboru Hanaoka, Koji Higashino, Noriya Uedo, \\ Ryu Ishihara, Hiroyasu lishi

\section{Institutions} \\ Department of Gastrointestinal Oncology, Osaka Medical Center for \\ Cancer and Cardiovascular Diseases, Osaka, Japan
}

submitted 27.7.2016

accepted after revision 24.10 .2016

Bibliography

DOI http://dx.doi.org/10.1055/s-0042-121002 |

Endoscopy International Open ; : E11-E16

(c) Georg Thieme Verlag KG Stuttgart · New York

ISSN 2364-3722

\section{Corresponding author}

Yoji Takeuchi, MD, Department of Gastrointestinal Oncology, Osaka

Medical Center for Cancer and Cardiovascular Diseases, 1-3-3

Nakamichi Higashinari-ku, Osaka 537-8511, Japan

Fax: +81-6-6981-4067

takeuti-yo@mc.pref.osaka.jp

\begin{abstract}
Background and study aims Complete closure of large mucosal defects after colorectal endoscopic submucosal dissection (C-ESD) is considered impossible in most cases because of the limited width of the open clip. We therefore invented a simple closure technique using clip-and-line, named "line-assisted complete closure (LACC)", and assessed its technical feasibility.

Patients and methods Between January and February 2016, we performed LACC in 11 patients after C-ESD and included them in this retrospective feasibility study. Outcome measures were procedural success rate, procedure time, and post-procedural complications.

Results The median size of the resected specimen was $36 \mathrm{~mm}$ (range $30-72 \mathrm{~mm}$ ). Procedural success was achieved in 10 of 11 cases $(91 \%)$. Those 10 cases required a median of 9 endoclips (range 6-12) for complete closure. Median procedure time for LACC was 14 minutes (range 6-22). No complications were observed in any of the cases after the procedure.

Conclusion LACC is a simple and feasible technique for complete closure of large mucosal defects after C-ESD.
\end{abstract}

\section{Introduction}

Colorectal endoscopic submucosal dissection (C-ESD) is a promising, minimally invasive treatment developed as an alternative to surgery. It allows removal of large superficial colorectal tumors en bloc. However, this may result in the creation of a large mucosal defect, which sometimes causes adverse events (AEs), such as bleeding, perforation, and electrocoagulation syndrome [1,2]. Preventing those AEs is important for improving the safety and applicability of C-ESD. Complete closure of such artificial mucosal defects has been reported to reduce those complications after colorectal endoscopic resection [3, 4]. However, complete closure of large mucosal defects after C-ESD is considered impossible in most cases because of the limited width of the open clip. We therefore invented a simple closure technique using clip-and-line, named "line-assisted complete closure (LACC)" [5]. Our idea was inspired by a traction technique performed during C-ESD using clip-and-line [6, 7]. We herein describe the details of our closure technique, and assess the technical feasibility of the method.

\section{Patients and methods}

\section{Patients}

Between January and February 2016, 28 patients underwent CESD at Osaka Medical Center for Cancer and Cardiovascular Diseases. Of these, 11 cases were performed or supervised by 1 endoscopic specialist (Y. T.), who invented LACC technique, and all the consecutive 11 cases underwent LACC after C-ESD and were included in this retrospective feasibility study. Outcome measures were procedural success rate, procedure time, and post-procedural complications, such as bleeding, perforation, and electrocoagulation syndrome. Procedural success was defined as complete closure of the mucosal defect after CESD, which means covering the mucosal defect with normal mucosa so that the submucosal layer cannot be seen. Procedure time was measured from insertion of the first clip-andline until achieving or giving up complete closure. Post-ESD bleeding was defined as bleeding requiring emergency endoscopic hemostasis or transfusion, or as a decrease in the hemo- 


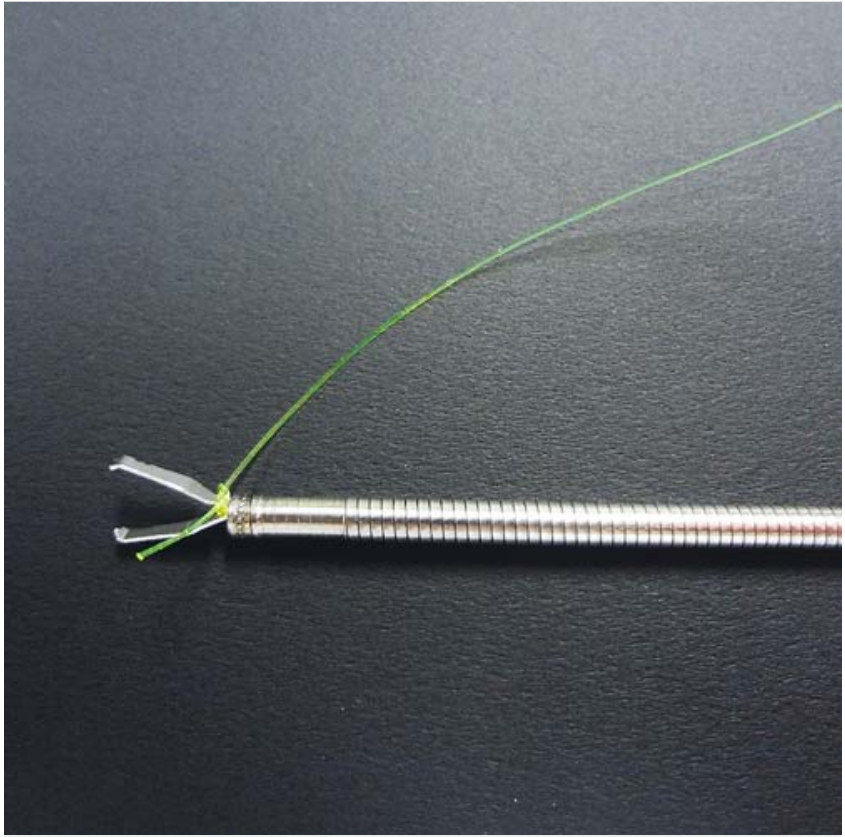

Fig. 1 A long nylon line was tied to the arm of an endoclip.
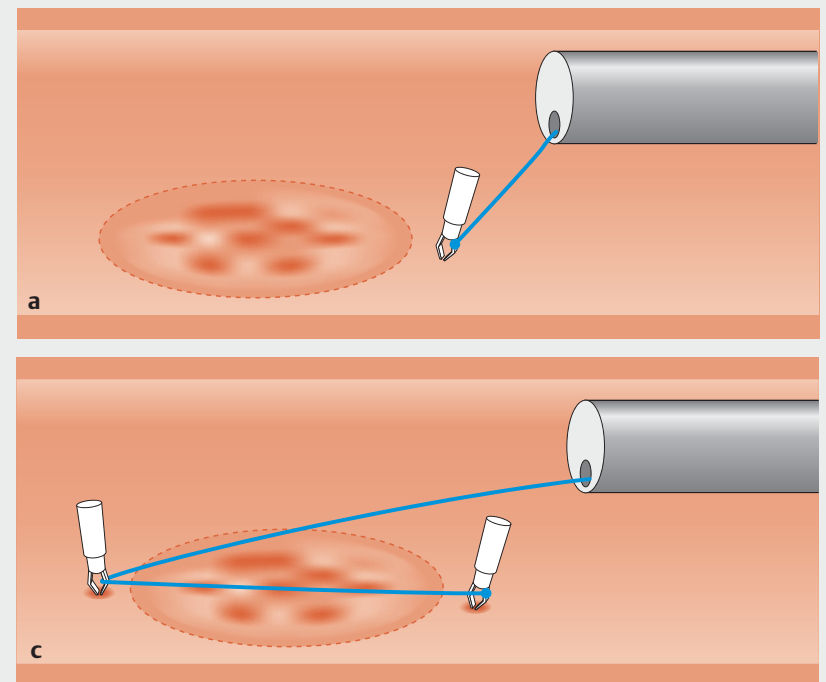

c

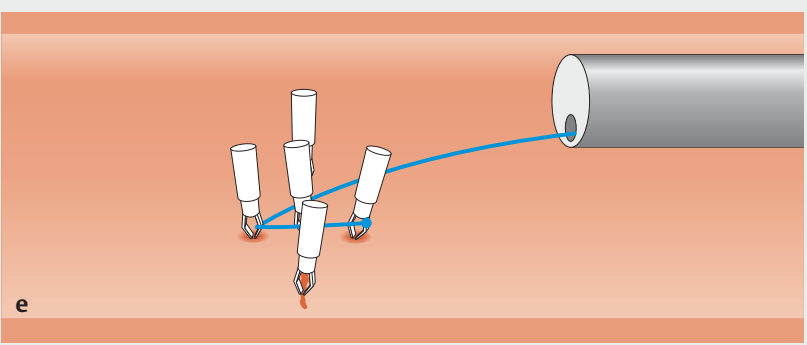

globin level of more than $2 \mathrm{~g} / \mathrm{dL}$ following C-ESD. Post-ESD perforation was defined as no perforation during the C-ESD procedure, followed by sudden appearance of symptoms of peritoneal irritation after the procedure, with the presence of free air on abdominal computed tomography (CT) or X-ray. A diagnosis of post-ESD electrocoagulation syndrome (PECS) was made if the patients presented with localized abdominal pain and fever $\left(\geq 37.6^{\circ} \mathrm{C}\right)$ or inflammatory response (leukocytosis $[\geq 10,000$ cells $/ \mu \mathrm{L}]$, increased C-reactive protein $[\geq 0.5 \mathrm{mg} / \mathrm{dl}]$ ), after C-ESD in the absence of visualized perforation on abdominal CT. This study was approved by the ethics committee at Osaka Medical Center for Cancer and Cardiovascular Diseases.

\section{Line-assisted complete closure (LACC)}

The LACC procedure was conducted immediately after completion of C-ESD using a colonoscope (PCF-Q260AZI, PCF-Q260JI, or CF-Q260DI; Olympus, Tokyo, Japan) with a distal attachment (D-201-13404 or D-201-11804; Olympus), without withdrawal and reinsertion of the colonoscope. The LACC procedure was performed in a uniform, standardized fashion as we previously described ( $\triangleright$ Fig. 1, $>$ Fig. 2, $\triangleright$ Fig.3, $>$ Video 1) [5]. At first, a long nylon line (e.g. a fishing line) was tied to the teeth of an endoclip (HX-610-090; Olympus), which was attached to
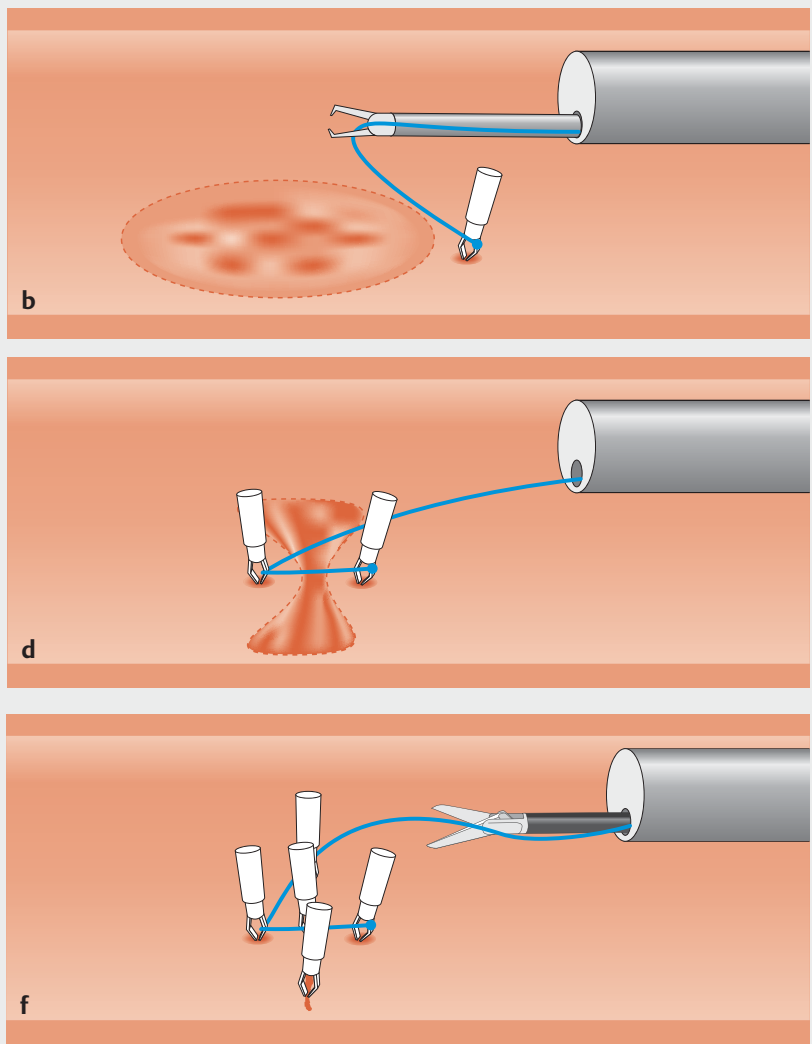

- Fig. 2 Schematic diagram of the line-assisted complete closure. a The endoclip and line were placed on the normal mucosa, $5 \mathrm{~mm}$ from the proximal margin of the wound. $\mathbf{b}$ Another endoclip without a line was then inserted into the accessory channel. $\mathbf{c}$ The line was anchored by another endoclip to the other side of the mucosa. $\mathbf{d}$ Both sides of the wound were gathered by pulling the anchored line. e Additional endoclips were placed to achieve complete closure. $f$ The line attached to the endoclip was cut using scissor forceps. 


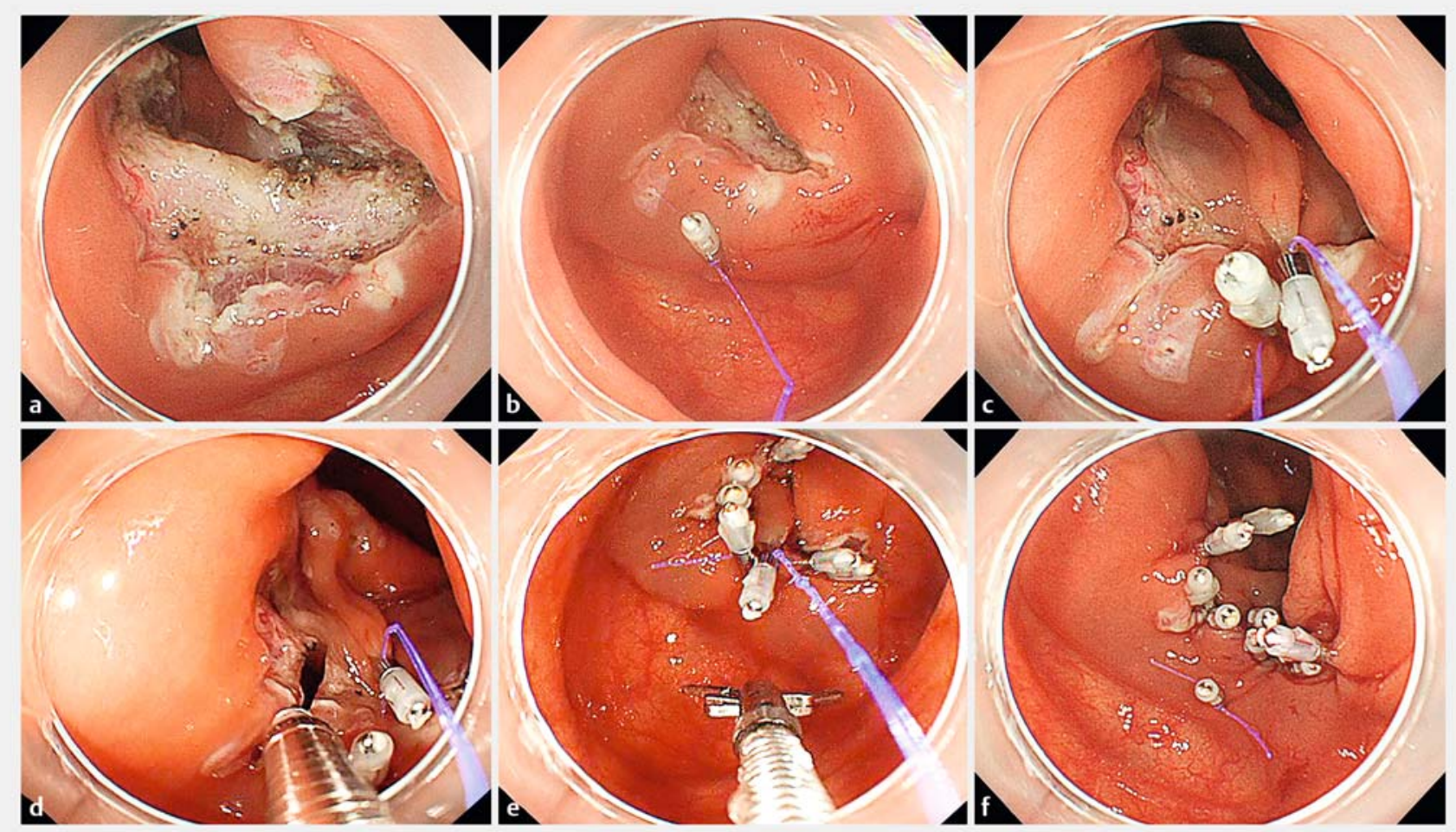

- Fig. 3 Endoscopic images of line-assisted complete closure. a A large mucosal defect after endoscopic submucosal dissection. b The endoclip and line were placed on the proximal margin of the wound. $\mathbf{c}$ The line was anchored by another endoclip to the other side of the mucosa, and both sides of the wound were gathered by pulling the anchored line. $\mathbf{d}$ Additional endoclips were placed to achieve complete closure. e, $\mathbf{f}$ The line attached to the endoclip was cut using scissor forceps.

an applicator (HX-110LR, Olympus; - Fig. 1). Based on a previous report, it was important not to open the endoclip fully at this moment [6]. The endoclip-and-line was then retracted into the applicator, and the applicator was inserted into the accessory channel. The endoclip-and-line was then placed on the normal mucosa, $5 \mathrm{~mm}$ from the proximal margin of the wound ( $\vee$ Fig.2a). Another endoclip without a line was then inserted through the accessory channel ( $\nabla$ Fig. $\mathbf{2} \mathbf{b}$ ) and anchored to the

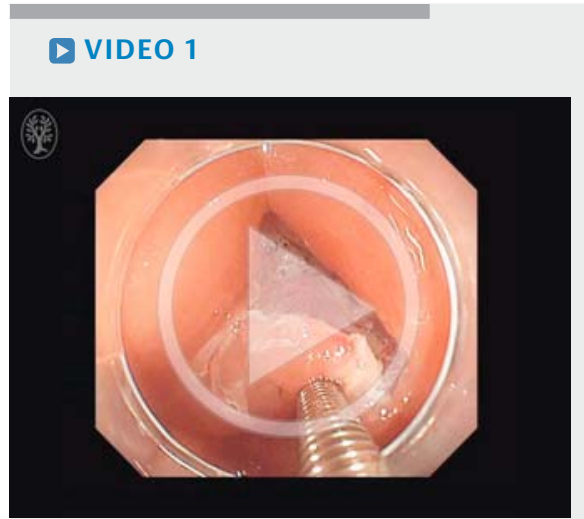

- Video 1: Line-assisted complete closure technique for a large mucosal defect after endoscopic submucosal dissection. other side of the normal mucosa ( $\triangleright$ Fig. 2 c). Both sides of the wound were gathered by gently pulling the anchored line ( $\triangleright$ Fig. 2 d). Additional endoclips were placed to achieve complete closure of the wound ( $\mathbf{F i g} . \mathbf{2 e}$ ). Multiple endoclip-andlines can be applied on demand, because LACC does not require withdrawal and reintroduction of a colonoscope. Only 1 assistant is needed to hold the line while clipping. The line tied to the endoclip was finally cut using scissor forceps (FS-3L-1; Olympus, $>$ Fig. 2f).

\section{Results}

Patient characteristics and outcomes of LACC are shown in - Table 1. Two lesions were located in the cecum, 4 in the ascending colon, 2 in the transverse colon, 2 in the sigmoid colon, and 1 in the rectum. C-ESD was successfully accomplished without perforation in all cases. Median size of the resected specimen was $36 \mathrm{~mm}$ (range 30-72). Procedural success was achieved in 10 of 11 cases ( $91 \%)$. These 10 cases required a median of 9 endoclips (range 6 -12) for complete closure. Median procedure time for LACC was 14 minutes (range 6-22). No complications after the procedure were observed in any of the cases. LACC failed in 1 case, in which the lesion was located at a flexure of the sigmoid colon. 

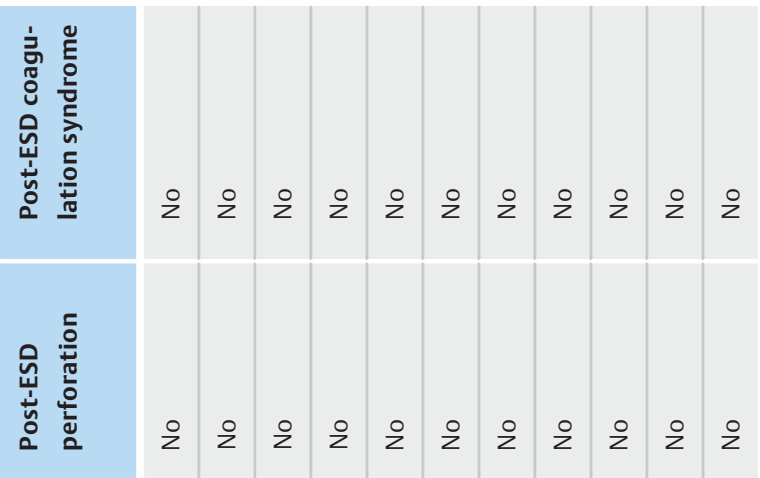

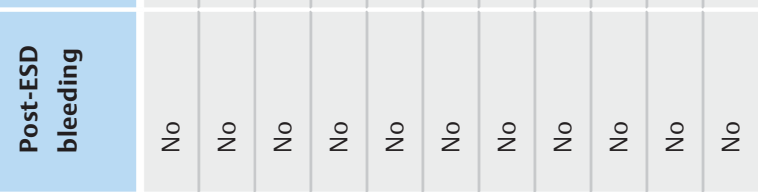
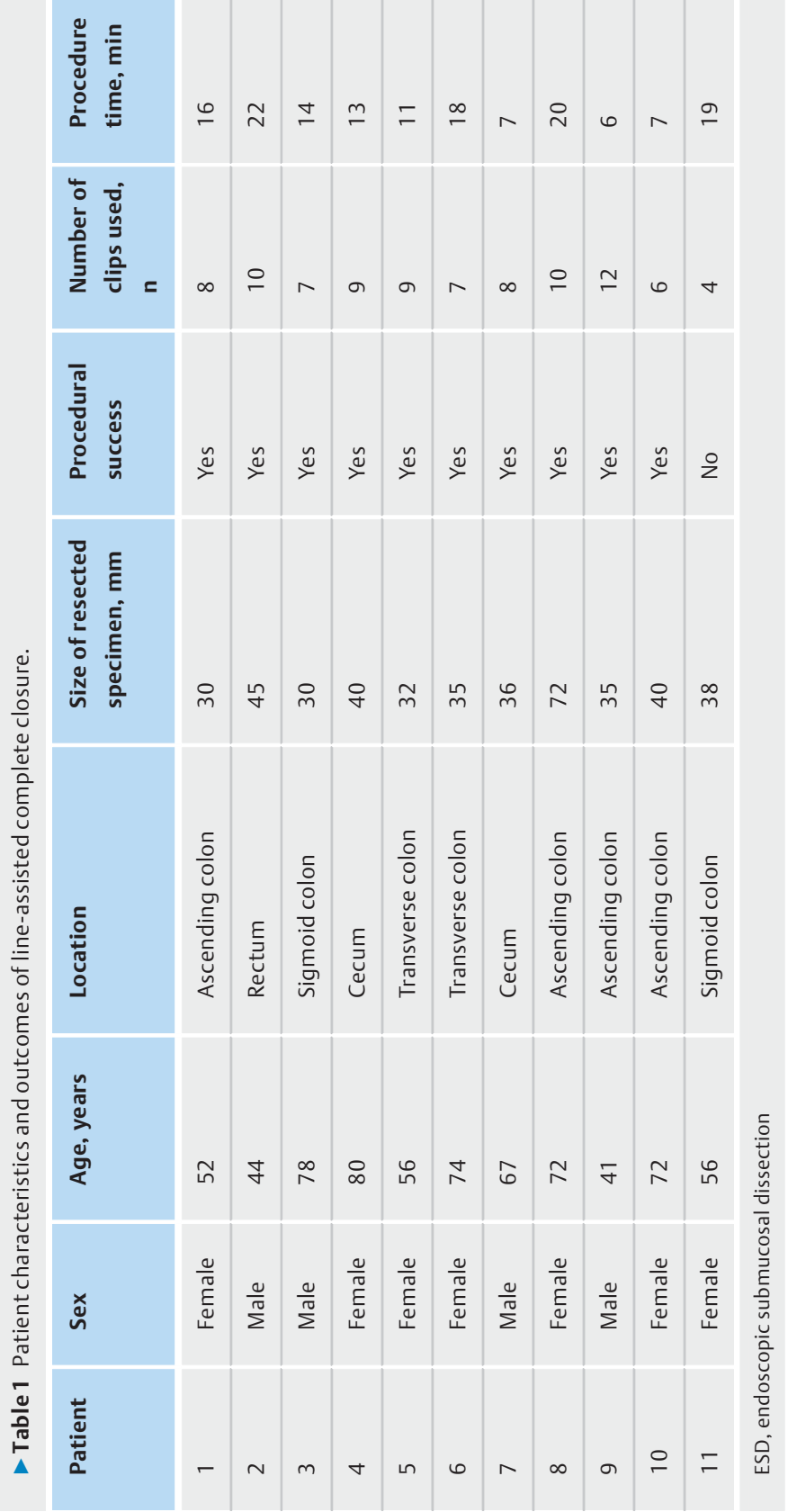


\section{Discussion}

In the current study, our newly developed closure technique, LACC, was successfully carried out on most of the patients with an acceptable procedure time. We could manage to close a large mucosal defect of up to $70 \mathrm{~mm}$ in diameter using this technique. Our results indicate that LACC is a feasible technique for closing large mucosal defects after C-ESD.

Various clip closure techniques have been reported to date, for example, a method using the "8-ring" [8], and techniques using clips and line named "loop clip" $[9,10]$ and "slip knot clip suturing" [11]. In contrast with our technique, the "8ring" technique requires the special device which is not always available. The "loop clip" technique requires only clips and line, which is the same as our technique; however, it needs one more step to make a nylon loop for preparation compared with our simple technique. In addition, because our technique can pull the line, we can change a field of vision for additional clipping. The "slip knot clip suturing" method is similar to our technique; however, making a slip knot loop is a complicated and timeconsuming process compared with our way of clip-and-line preparation ( $\mathbf{F i g . 1}$ ). The authors also reported a simpler technique later, which is very similar to our technique, almost at the same time we first reported about LACC $[5,12]$. As the later report was just a case report and their outcomes were never disclosed, we consider that this is the first report to assess the feasibility of this simple closure technique.

The over-the-scope clip (OTSC) system (Ovesco Endoscopy, Tübingen, Germany) is now commercially available and may be another option for closing large artificial mucosal defects of the gastrointestinal tract $[13,14]$. However, it is not always applicable to colorectal lesions because to apply this method, the colonoscope needs to be withdrawn after C-ESD to mount the applicator cap of the OTSC system. In contrast, LACC technique does not require withdrawal and reinsertion of the colonoscope after C-ESD, which is the biggest advantage of LACC, especially for proximal colonic lesions or patients with difficult colonoscopic intubation.

In this study, we successfully closed all the mucosal defects in proximal colon ( 2 in cecum and 4 in ascending colon), and there were no technical difficulties in performing LACC in such locations. However, we failed to achieve complete closure in 1 case, in which the lesion was located at the flexure of the sigmoid colon. This was because the maneuverability and stability of the colonoscope were poor in that particularly narrow and winding location of the colon. In the failed case, instability of the colonoscope caused too much traction to the clip-and-line device, which consequently result in clip detachment. Applying a shielding method using polyglycolic acid sheets (Neoveil; Gunze Co., Kyoto, Japan) with fibrin glue (Beri-plast P 3-mL Combi-Set; CSL Behring Pharma, Tokyo, Japan) might be easier than clip closure in such difficult locations $[15,16]$. Our method achieves a tight closure by gathering the mucosa directly by pulling the line, even in cases in the rectum, which is thought to have less wall mobility. Of course, the procedure for a large lesion was a bit difficult and required more time. However, by using multiple clip-and-line devices, we also managed to close a 40-mm mucosal defect resulting from gastric ESD [17].

We did not experience any post-ESD complications in the 10 patients in whom we achieved complete closure of the large mucosal defect. Although some studies showed that complete closure of large artificial mucosal defects reduces complications after colorectal endoscopic resection [3, 4], further investigation is required to clarify whether complete closure really results in a clinical benefit. We previously reported that rates of delayed perforation and bleeding after C-ESD were not high ( $1.0 \%$ and $3.0 \%$, respectively), however, incidence of PECS, which required a longer fasting period and hospital stay after C-ESD, was $9.5 \%$ [2]. Therefore, we consider that preventing PECS is an important issue, and complete closure by LACC has the potential to solve the problem. Of course, taking into account cost-effectiveness, it might be reasonable to apply the technique only to high-risk cases for PECS, such as female patients with large tumor located in the ascending colon or cecum [2]. In fact, we have started a prospective, randomized controlled trial to elucidate whether LACC is effective in reducing incidence of PECS in such a high-risk population.

This feasibility trial is limited by its small sample size and retrospective design; thus, we cannot state that it yielded clinical benefits at the moment. However, the high procedural success rate and relatively short procedure time showed its technical feasibility. Also, the optimal line for this technique should be considered. LACC requires additional clipping through an endoscopic channel with an inserted line for clip-and-line device. Because a thick line would disturb repeat insertion of the endoclip applicator, dental floss is not an optimal line for LACC. Because the line used in LACC does not suture colonic mucosa directly, we believe that a fishing line would be acceptable, but using a surgical suture thread would warrant the safety of the procedure.

\section{Conclusion}

In conclusion, LACC is feasible for complete closure of large mucosal defects after C-ESD. We consider that this simple method is valuable and should be further assessed to determine its clinical benefit.

\section{Competing interests}

None

\section{References}

[1] Takeuchi Y, lishi H, Tanaka S et al. Factors associated with technical difficulties and adverse events of colorectal endoscopic submucosal dissection: retrospective exploratory factor analysis of a multicenter prospective cohort. Int J Colorectal Dis 2014; 29: 1275-1284

[2] Yamashina T, Takeuchi Y, Uedo $\mathrm{N}$ et al. Features of electrocoagulation syndrome after endoscopic submucosal dissection for colorectal neoplasm. J Gastroenterol Hepatol 2016; 31: 615-620 
[3] Zhang QS, Han B, Xu JH et al. Clip closure of defect after endoscopic resection in patients with larger colorectal tumors decreased the adverse events. Gastrointest. Endosc 2015; 82: 904 - 909

[4] Fujihara S, Mori H, Kobara $\mathrm{H}$ et al. The efficacy and safety of prophylactic closure for a large mucosal defect after colorectal endoscopic submucosal dissection. Oncol Rep 2013; 30: 85-90

[5] Kato M, Takeuchi Y, Yamasaki Y. Line-assisted complete closure of a large colorectal mucosal defect after endoscopic submucosal dissection. Dig Endosc 2016; 28: 686

[6] Yamasaki Y, Takeuchi Y, Hanaoka $\mathrm{N}$ et al. A novel traction method using an endoclip attached to a nylon string during colonic endoscopic submucosal dissection. Endoscopy 2015; 47: E238-E239

[7] Suzuki S, Gotoda T, Kobayashi Y et al. Usefulness of a traction method using dental floss and a hemoclip for gastric endoscopic submucosal dissection: a propensity score matching analysis (with videos). Gastorointest Endosc 2015; 83: 337 - 346

[8] Fujii T, Ono A, Fu K. A novel endoscopic suturing technique using a specially designed so-called "8-ring" in combination with resolution clips (with videos). Gastrointes Endosc 2007; 66: 1215-1220

[9] Sakamoto N, Beppu K, Matsumoto K et al. “Loop Clip”, a new closure device for large mucosal defects after EMR and ESD. Endoscopy 2008; 40: E97-E98

[10] Mori H, Sakamoto N, Osada T et al. The "Loop clip" is useful for closing large mucosal defects after colorectal endoscopic submucosal dissection: a preliminary clinical study. Dig Endosc 2011; 23: 330 - 331
[11] Nishizawa T, Uraoka T, Sagara S et al. Endoscopic slipknot clip suturing method: an ex vivo feasibility study (with video). Gastrointest. Endosc 2016; 83: 447 - 450

[12] Yahagi N, Nishizawa T, Akimoto T et al. New endoscopic suturing method: "String clip suturing method". Gastrointest Endosc 2016; 84: $1064-1065$

[13] Schurr MO, Hartmann C, Ho CN et al. An over-the-scope clip (OTSC) system for closure of iatrogenic colon perforations: results of an experimental survival study in pigs. Endoscopy 2008; 40: 584- 588

[14] Maekawa S, Nomura R, Murase T et al. Complete closure of artificial gastric ulcer after endoscopic submucosal dissection by combined use of single over-the-scope clip and through-the-scope clips (with videos). Surg Endosc 2015; 29: 500 - 504

[15] Takimoto K, Toyonaga T, Matsuyama K. Endoscopic tissue shielding to prevent delayed perforation associated with endoscopic submucosal dissection for duodenal neoplasms. Endoscopy 2012; 44: E414-415

[16] Tsuji Y, Ohata K, Gunji T et al. Endoscopic tissue shielding method with polyglycolic acid sheets and fibrin glue to cover wounds after colorectal endoscopic submucosal dissection (with video). Gastrointest Endosc 2014; 79: 151 - 155

[17] Yamasaki Y, Takeuchi Y, Kato M et al. Line-assisted complete closure of large gastric mucosal defect using multiple clip-and-line technique. VideoGIE 2016; 1: 49-50 